\title{
Teaching Emergency Surgical Skills for Trauma Resuscitation-Mechanical Simulator versus Animal Model
}

\author{
Jameel Ali, Anne Sorvari, and Anand Pandya \\ Department of Surgery, St. Michael's Hospital, University of Toronto, 55 Queen Street East, \\ Suite No. 402, Toronto, ON, Canada M5C 1R6 \\ Correspondence should be addressed to Jameel Ali, alij@smh.ca
}

Received 8 August 2012; Accepted 27 August 2012

Academic Editors: A. K. Attri, D. Doll, A. Eisenman, and M. Mohsin

Copyright ( $) 2012$ Jameel Ali et al. This is an open access article distributed under the Creative Commons Attribution License, which permits unrestricted use, distribution, and reproduction in any medium, provided the original work is properly cited.

Background. Traditionally, surgical skills in trauma resuscitation have been taught using animal models in the advanced trauma life support (ATLS) course. We compare one mechanical model (TraumaMan simulator) as an alternative teaching tool for these skills. Method. Eighteen providers and 14 instructors performed four surgical procedures on TraumaMan and compared educational effectiveness with the porcine model. Evaluation was conducted (Likert system 1: very poor to 5: excellent). The participants indicated if TraumaMan was a suitable (scale 1: not suitable to 4: excellent) ATLS teaching model considering cost, animal ethics concerns, and other factors. Comments were solicited for both models. Results. Overall ratings for educational effectiveness of the 4 skills ranged from 3.58 to 4.36 for the porcine and 3.48 to 4.29 for the TraumaMan model. TraumaMan suitability was rated 3-4 (mean 3.38) by $84 \%$ participants. TraumaMan as a substitute for the porcine model was recommended by $85 \%$ participants. With no ethical or cost concerns, $44 \%$ students and $71 \%$ instructors preferred TraumaMan. Considering all factors, TraumaMan was preferred by $78 \%$ students and $93 \%$ instructors. Conclusions. TraumaMan is a suitable alternative to the porcine model and considering all factors it may be the preferred method for teaching ATLS emergency trauma surgical skills.

\section{Introduction}

Resuscitation of critically injured patients is conducted primarily in the emergency room setting. The surgical resuscitation skills have been taught internationally through the Advanced Trauma Life Support (ATLS) Course of the American college of Surgeons. This course was introduced in Nebraska in the 1970s [1] and adopted by American College of Surgeons (ACS) in an effort to standardize and improve management of trauma patients. Since then, more than a million doctors in 62 countries have completed this course, which consists of lectures, group discussions, standardized patients, and technical skill stations. An important component of the ATLS program is the surgical skills station which provides hands-on training in performing procedures such as cricothyroidotomy, chest tube insertion, pericardiocentesis, and diagnostic peritoneal lavage (DPL). Logistic, societal, and economic issues have prompted the development of alternatives to the animal model including human patient simulators (HPS) in teaching the surgical skills component.

The use of simulation-based technology in trauma education has provided a means for enhancing patient safety, reducing medical errors, and performing systematic evaluation of various competencies [2-4]. The American College of Surgeons has approved the TraumaMan (Simulab Corporation, Seattle, WA, USA) along with other models as alternatives for teaching ATLS surgical skills after evaluation by members of the ATLS subcommittee. The TraumaMan system consists of a mechanical model with simulated human tissue structure designed specifically to mimic the surgical dissection of the thoracic, abdominal, and neck regions for teaching the ATLS surgical skills. Due to ethical concerns surrounding the use of animals for teaching and 
(1) For the surgical procedures please rate the educational effectiveness of the model in learning the skill using the following Likert Scale $(1=$ very poor, $2=$ poor,

3 = satisfactory, 4 = very good, 5 = excellent)

(2) Recognizing the concerns for using animal models for learning surgical skills and the need to find a nonanimal model, please rate the degree to which you think the TraumaMan is a suitable model for learning surgical skills in the ATLS course. Please use the following Likert scale ( 1 = not suitable, $2=$ acceptable, 3 = very acceptable, 4 = excellent)

(3) Would you recommend the TraumaMan as a substitute for the animal model? (Yes, Neutral, No)

(4) Overall, if there were no ethical or animal model concerns, which model will you recommend?

(5) Overall, considering all factors (animal concerns, suitability of the models, financial, etc.) which model would you recommend?

Box 1: Survey to compare the effectiveness of the porcine model versus TraumaMan for teaching ATLS surgical skills.

the availability of accredited alternatives, more than two hundred ATLS course centers in the United States have adopted human patient simulators for teaching surgical skills. However, this alternative has not been uniformly accepted [5]. Many, including those who feel compelled to use mechanical models because of animal ethics concerns, have questioned their suitability for teaching the resuscitation surgical skills. An unbiased comparison of the mechanical and animal models seems warranted. Our experience with the porcine model has been excellent. However, animal ethics approval for the use of animal teaching models requires assurance that no alternative nonanimal model is suitable for this purpose. We therefore embarked on a study to determine the suitability of one of the available mechanical models for surgical skills teaching in the ATLS course-TraumaMan.

\section{Materials and Methods}

ATLS surgeon instructors and surgical residents who had previously taught or completed our ATLS courses using the porcine model were openly invited to assess the TraumaMan simulator. After a brief orientation, all participants performed the necessary surgical skills of cricothyroidotomy, chest tube insertion, pericardiocentesis, and diagnostic peritoneal lavage. Following the practicum, participants completed a feedback questionnaire covering 5 components as indicated in Box 1. A Likert scale of 1: very poor to 5: excellent was used for educational effectiveness. Suitability was rated on a 4-point Likert scale of 1: not suitable to 4: excellent. Items 3-5 in Box 1 were completed as indicated. Participants were also invited to make general comments on the advantages and disadvantages of both models and these were reviewed.

The mean and standard deviations of the Likert scores were calculated and between-group comparisons conducted using unpaired $t$-tests. Model preferences were compared by Fischer Exact test. A $P$ value of $\leq 0.05$ was regarded as statistically significant.

\section{Results}

Thirty-two participants (18 students, 14 ATLS instructors) evaluated ATLS surgical skills on the TraumaMan model and compared this to previous experience using the animal model. Please note that in the tables the numbers do not always add up to 32 because of variation in the number of responses received for these items.

Table 1 shows the survey results for all participants on the educational effectiveness of the porcine and TraumaMan models with the mean ratings out of a maximum of 5. For chest tube insertion, 28 participants in the porcine model and 26 in the TraumaMan model rated 4 or 5 . Corresponding cricothyroidotomy ratings of 4 to 5 were from 27 participants for TraumaMan and 16 for the porcine model. Corresponding ratings of 4-5 for DPL came from 25 participants for the porcine model and 15 in TraumaMan. For pericardiocentesis a rating of 4-5 was assigned by 20 participants in the porcine model and 26 in the TraumaMan model. Mean (SD \pm ) Likert scores for each procedure are also shown in Table 1. Also shown are the $P$ values for comparing each of those procedures between TraumaMan and the porcine model, where it is seen that TraumaMan had higher scores in two procedures (pericardiocentesis, $P=0.0195$, and cricothyroidotomy, $P=0.0010)$, porcine model higher in one procedure (DPL, $P=0.0089)$, and no statistically significant difference with the chest tube $(P=0.3759)$.

Table 2 shows that, considering animal ethical concerns, overall $84 \%$ of participants considered TraumaMan to be very acceptable to excellent as a teaching model for the ATLS surgical skills with an overall mean rating of 3.38 out of 4 . Also, Table 2 shows the $P$ value of 0.079 for the comparison between TraumaMan and the porcine model.

As indicated in Table 3, TraumaMan was recommended as a substitute for the porcine model by $85 \%$ of participants and there was no statistically significant difference between the recommendations between students and teachers $(P=$ 0.3547, by Fisher Exact Test). Comparing yes to no/neutral resulted in a $P$ value of 0.0043 for recommending Traumaman as indicated in Table 3. 
TABLE 1: Survey scores of the porcine versus TraumaMan for educational effectiveness of the surgical skills.

\begin{tabular}{|c|c|c|c|c|c|}
\hline & $\begin{array}{c}\text { Very Poor } \\
1\end{array}$ & $\begin{array}{c}\text { Poor } \\
2\end{array}$ & $\begin{array}{c}\text { Satisfactory } \\
3\end{array}$ & $\begin{array}{c}\text { Very good } \\
4\end{array}$ & $\begin{array}{c}\text { Excellent } \\
5\end{array}$ \\
\hline \multicolumn{6}{|c|}{ ATLS surgical skills: porcine model scores } \\
\hline Chest tube & 0 & 0 & $13 \%(4)$ & $41 \%(13)$ & $47 \%(15)$ \\
\hline Pericardiocentesis & 0 & $7 \%(2)$ & $27 \%(8)$ & $53 \%(16)$ & $13 \%(4)$ \\
\hline DPL & 0 & 0 & $19 \%(6)$ & $58 \%(18)$ & $23 \%(7)$ \\
\hline Cricothyroidotomy & 0 & $6 \%(2)$ & $44 \%(14)$ & $34 \%(11)$ & $16 \%(5)$ \\
\hline \multicolumn{6}{|c|}{ ATLS surgical skills: TraumaMan scores } \\
\hline Chest tube & 0 & $3 \%(1)$ & $16 \%(5)$ & $41 \%(13)$ & $41 \%(13)$ \\
\hline Pericardiocentesis & 0 & 0 & $19 \%(6)$ & $66 \%(21)$ & $16 \%(5)$ \\
\hline DPL & 0 & 0 & $52 \%(16)$ & $39 \%(12)$ & $10 \%(3)$ \\
\hline \multirow[t]{2}{*}{ Cricothyroidotomy } & 0 & $3 \%(1)$ & $13 \%(4)$ & $38 \%(12)$ & $47 \%(15)$ \\
\hline & \multicolumn{2}{|c|}{ Porcine } & TraumaMan & \multicolumn{2}{|c|}{$P$ value } \\
\hline Chest tube & \multicolumn{2}{|c|}{$4.36(\mathrm{SD} \pm 0.70)$} & $4.19(\mathrm{SD} \pm 0.82)$ & \multicolumn{2}{|c|}{$P=0.3759$} \\
\hline Cricothyroidotomy & \multicolumn{2}{|c|}{$3.58(\mathrm{SD} \pm 0.84)$} & $4.29(\mathrm{SD} \pm 0.81)$ & \multicolumn{2}{|c|}{$P=0.0010$} \\
\hline DPL & \multicolumn{2}{|c|}{$3.94(\mathrm{SD} \pm 0.67)$} & $3.48(\mathrm{SD} \pm 0.67)$ & \multicolumn{2}{|c|}{$P=0.0089$} \\
\hline Pericardiocentesis & \multicolumn{2}{|c|}{$3.55(\mathrm{SD} \pm 0.78)$} & $3.97(\mathrm{SD} \pm 0.59)$ & \multicolumn{2}{|c|}{$P=0.0195$} \\
\hline
\end{tabular}

TABLE 2: Suitability for learning surgical skills in the ATLS course with TraumaMan recognizing the concerns of animal models.

\begin{tabular}{|c|c|c|c|c|c|c|}
\hline \multirow{2}{*}{ Participants } & \multicolumn{4}{|c|}{ Scores } & \multirow{2}{*}{ Mean score } & \\
\hline & 1 & 2 & 3 & 4 & & \\
\hline Student $(n=18)$ & 0 & 4 & 7 & 7 & $3.17(\mathrm{SD} \pm 0.79)$ & \multirow{2}{*}{$P$ value $=0.079$} \\
\hline Teacher $(n=14)$ & 0 & 1 & 3 & 10 & $3.64(\mathrm{SD} \pm 0.63)$ & \\
\hline Overall $(n=32)$ & 0 & $5(16 \%)$ & $10(31 \%)$ & $17(53 \%)$ & $3.38(\mathrm{SD} \pm 0.75)$ & \\
\hline
\end{tabular}

1: Not suitable, 2: acceptable, 3: very acceptable, and 4: excellent.

TABle 3: Recommend TraumaMan as a substitute for the porcine model.

\begin{tabular}{lccc}
\hline & -1 & 0 & 1 \\
& No & Neutral & Yes \\
\hline Student $(n=18)$ & 2 & 2 & 14 \\
Teacher $(n=14)$ & 0 & 1 & 13 \\
\hline Overall $(n=32)$ & $2(6 \%)$ & $3(9 \%)$ & $27(85 \%)$ \\
\hline
\end{tabular}

Fisher Exact Test comparing No/Neutral to Yes, $P=0.0043$.

Table 4 shows that if there were no ethical or animal model concerns, then overall $56 \%$ of participants would recommend the use of TraumaMan versus $44 \%$ who would prefer the use of the porcine model for ATLS training. However, $71 \%$ of instructors versus $44 \%$ of students prefer TraumaMan. $P$ value by Fisher Exact Test between students and instructors was 0.0002 .

Table 5 demonstrates that participants overwhelmingly (93\% instructors and 78\% students) support the use of TraumaMan for training in ATLS surgical skills while considering all factors (ethical, financial, portability and suitability for learning), $(P=0.0043$ by Fisher Exact Test $)$. However, there was a clear difference in preference for TraumaMan between instructors (71\% with no concerns and 93\% considering all factors) and students (44\% with no concerns and $78 \%$ considering all factors).
From the feedback provided in the comments (detailed in Table 6), participants preferred the animal model due to more realistic surgical handling, dynamic tissue changes, and good anatomical detail. The disadvantage of the animal model included the ethical dilemma related to sacrifice of the animal, the inability to repeatedly practice on the same animal, and the requirement for an animal care facility. Furthermore the airway anatomy in the porcine model was not similar to humans and the cricothyroidotomy procedure could only be performed once on the animal model.

In contrast, the advantages of TraumaMan included the absence of ethical concerns, ability to repeat procedures consistently on the model, the portability and availability of the model without the need for special vivarium facilities, and the opportunity for all participants to practice all the surgical skills especially cricothyroidotomy. The disadvantages of the TraumaMan model included the initial capital cost and the brittle rubbery feel of the tissues especially while inserting the chest tube. However, many participants indicated that the unrealistic feel was less important to teaching compared to the ethical dilemma of animal use.

\section{Discussion}

The use of simulation to educate and train physicians has been gaining increasing attention since the Institute of Medicine issued its 1999 report, "To Err is Human" [6]. 
TABLE 4: Preference for training in ATLS surgical skills if there were no ethical or porcine model considerations.

\begin{tabular}{lccc}
\hline Participants & Porcine model $(\%, n)$ & TraumaMan preference $(\%, n)$ & Fisher Exact Test comparing students with teachers \\
\hline Student $(n=18)$ & $56 \%(10)$ & $44 \%(8)$ & $P=0.0002$ \\
Teacher $(n=14)$ & $29 \%(4)$ & $71 \%(10)$ & \\
\hline Overall $(n=32)$ & $44 \%(14)$ & $56 \%(18)$ & \\
\hline
\end{tabular}

TABLE 5: Preference for training in ATLS surgical skills considering all factors.

\begin{tabular}{lcc}
\hline Participants & Porcine Model $(\%, n)$ & TraumaMan preference $(\%, n)$ \\
\hline Student $(n=18)$ & $22 \%(4)$ & $78 \%(14)$ \\
Teacher $(n=14)$ & $7 \%(1)$ & $93 \%(13)$ \\
Overall $(n=32)$ & $16 \%(5)$ & $84 \%(27)$ \\
\hline
\end{tabular}

$P=0.0043$ by Fisher Exact Test comparing TraumaMan to the porcine model.

The Institute of Medicine report suggested that innovative approaches to education and the use of simulation technology may help to reduce the projected 98,000 deaths that occur each year in the United States because of medical errors. As a result, simulation technology has now been embraced by a variety of specialties as an advanced educational tool for training and evaluation of clinical performance $[7,8]$. In order for simulators to be effective, it is important to overcome three potential challenges: (1) realism: organ responses, physiologic variables, tissue-tool interfaces, visual displays, and real-time interactions should be as realistic to the user as possible; (2) authenticity: simulation technology should be easily integrated into educational curricula, provide training that is clinically useful, and allow for validated performance metrics; (3) acceptability: commitment to simulated testing in medical centers, acceptance by teaching faculty, and approval and support by medical specialty organizations such as the American Association for surgery of Trauma. In addition, provision of devices at an acceptable financial cost is also needed [9].

The use of simulation based technology in trauma education has focused on providing a safe and effective alternative to the more traditional methods that are used to teach technical skills and critical concepts in trauma resuscitation [10]. In recent years, TraumaMan has become widely used in ATLS as an adjunct to teach essential surgical skills. The utility of TraumaMan for ATLS education was investigated by Block et al. [11] in 2002. In their study, students found TraumaMan to be superior to traditional animal models for learning surgical airway skills and the management of pneumothorax. Berkenstadt et al. [12] in 2006 further evaluated TraumaMan as a training tool for chest drain insertion. According to the participants, the TraumaMan simulator was superior to the animal model only in teaching anatomical landmarks, while the animal model was superior in teaching tissue handling, dissection, and chest drain fixation. The present study differs from those previously reported in that we compared the training effectiveness and preferences of students and instructors for all four ATLS surgical skills in both the porcine and TraumaMan models in ATLS teaching. Considering the ethical issues related to animal use, TraumaMan was the overwhelmingly preferred model.
To meet the increasing demand for trauma-based education using simulation, a number of commercial simulators have been introduced to meet specific educational objectives. Airway management using the Air-Man simulator (Laerdal, Norway), High Fidelity Patient Simulator (METI), and the SimMan simulator have been used to teach airway management skills in trauma patients. The VIRGIL Chest Trauma Training System (the Simulation Group-CIMIT, Boston, MA) is used for the training of medics in combat casualty [13]. There is an ongoing need to selectively develop additional high-fidelity simulators and software for computerized instruction and innovative web-based teaching. As these technologies are developed, there must be a concerted effort to formally validate and demonstrate that these modalities are as good as, if not better than, traditional methods of trauma teaching. Convincing evidence is necessary if trauma-based simulation is to become a strong, viable, and universally accepted teaching adjunct.

A very important consideration in utilizing the animal model for ATLS training is the ethical dilemma of animal use when an alternative method is available. Surgeons have long practiced and learned their skills on live pigs because of their anatomical similarities to humans. In 2006, statistics from the Canadian Council on Animal Care show that 38,000 pigs were used for education [5]. The American College of Surgeons, which accredits the ATLS program, has approved the use of human patient simulators such as TraumaMan to teach the 4 procedures - chest tube insertion, pericardiocentesis, peritoneal lavage, and cricothyroidotomy. This approval, however, was not accompanied by formal comparison of these mechanical models with the animal model as is reported in our study. With the acceptance of the mechanical model as a suitable alternative for teaching the ATLS surgical skills, many animal ethics committees may not approve live animal use for teaching these skills. In addition, the preparation time and provision of live animal models add significant time commitment as well as cost to support an animal care facility. However, the feasibility of utilizing the TraumaMan model is limited by the number of models and the high cost for providing these models for student training ( $\$ 25,000$ USD per model). Once acquired, these mechanical models provide a continuing medium for clinical education [14]. 
TABLE 6: Summary of comments by participants.

\begin{tabular}{ll}
\hline Advantages & Disadvantages \\
\hline (i) More realistic feel and handling & Porcine model \\
$\begin{array}{ll}\text { (ii) More surgically realistic } & \text { (i) Ethical concerns_having to sacrifice the animal } \\
\text { (iii) Real-time live animal } & \text { (ii) Cannot repeatedly practice on the same animal } \\
\text { (iv) Moving tissues } & \text { (iii) Cricothyroidotomy can only be done once } \\
\text { (v) Anatomic detail is good_-representation of the layers of body wall } & \text { (iv) Expensive } \\
\text { (vi) Likely cheaper } & \\
\text { (vii) Presence of blood in operative procedure field } & \\
\text { (viii) Presence of active monitoring more like trauma bay not similar to human } \\
\text { (ix) Psychological factor of practicing on a living animal }\end{array}$
\end{tabular}

(ix) Psychological factor of practicing on a living animal

TraumaMan model
(i) Ethical advantage-no animal use
(i) Not real feel of human tissue
(ii) Procedures can be repeated many times
(ii) Not real feel to chest wall
(iii) Cost advantage versus animal
(iii) Cost of trauma man
(iv) Consistent anatomy
(iv) Bloodless field
(v) Easy to learn and practice
(v) Cricothyroidotomy space is larger than reality
(vi) Easy to teach
(vi) Tissues more brittle than human-chest wall is easy to enter
(vii) Always available for practice
(vii) Rubber poorly simulates skin
(viii) Good to learn landmarks—-similar to human anatomy
(viii) Needs better simulation of underlying tissue layers

(ix) Less smelly

(x) Reproducible technique

(xi) Opportunity for all participants to try all procedures

(xii) Portability_can be transported to another hospital

(xiii) No need for special animal laboratory space

(xiv) Reusable

Additional Comments

(i) Combined tissue/synthetic model may be more preferable.

(ii) Cricothyroidotomy needs a more elaborate anatomical kit with more details.

(iii) Chest wall is easy to enter. It may benefit from more thickness or layers in the TraumaMan model.

(iv) Unrealistic feel is less important to teaching compared to ethical dilemmas with animal use.

There are several limitations of this study. The size of the population studied was quite small. Conceivably increasing the statistical power and widening the Likert response scale may yield different results. One of the possible biases in this study may lay in participants' attitude towards the use of animals for training, which could change their attitude in favor of one of the training modalities. The participation in this study was voluntary and it is conceivable that only subjects interested in the respective models may have participated leading to possible bias.

In spite of these limitations, our study appears to have demonstrated the effectiveness of the TraumaMan simulator in teaching the surgical skills of the ATLS course. The TraumaMan model is perceived as providing better training with respect to surgical airway skills and pericardiocentesis. The TraumaMan model is also considered to be the preferred training tool given the ethical, financial, and resource constraints related to animal use.

As a result of our study, we have switched our surgical skills model in the ATLS course to TraumaMan because we could not justify identifying animals as the only suitable source for providing the necessary training in our ethics application for renewal. Conceivably other mechanical models may prove just as educationally effective but our study was limited to comparing TraumaMan with the porcine model and thus we are unable to comment on the other models which could also be suitable substitutes for the porcine model.

\section{References}

[1] P. E. Collicott and I. Hughes, "Training in advanced trauma life support," The Journal of the American Medical Association, vol. 243, no. 11, pp. 1156-1159, 1980.

[2] R. K. Reznick and H. MacRae, "Teaching surgical skillschanges in the wind," The New England Journal of Medicine, vol. 355, no. 25, pp. 2664-2669, 2006.

[3] J. Ali, R. U. Adam, I. Sammy, E. Ali, and J. I. Williams, "The simulated trauma patient teaching module-does it improve student performance?" Journal of Trauma-Injury, Infection and Critical Care, vol. 62, no. 6, pp. 1416-1420, 2007.

[4] M. M. Knudson and A. C. Sisley, "Training residents using simulation technology: experience with ultrasound for trauma," Journal of Trauma-Injury, Infection and Critical Care, vol. 48, no. 4, pp. 659-665, 2000. 
[5] R. Collier, "Switching swine for simulators: at what cost?" Canadian Medical Association Journal, vol. 179, no. 8, pp. 759$760,2008$.

[6] L. T. Kohn and J. M. Corrigan, Donaldson $m$ Sets, "To Err Is HuMan", Institute of Medicine, National Academy Press, Washington, DC, USA, 1999.

[7] J. Ali, T. J. Gana, and M. Howard, “Trauma mannequin assessment of management skills of surgical residents after advanced trauma life support training," Journal of Surgical Research, vol. 93, no. 1, pp. 197-200, 2000.

[8] J. Ali, R. J. Cohen, T. J. Gana, and K. F. Al-Bedah, "Effect of the advanced trauma life support program on medical student's performance in simulated trauma patient management," Journal of Trauma-Injury, Infection and Critical Care, vol. 44, no. 4, pp. 588-591, 1998.

[9] S. L. Dawson, "A critical approach to medical simulation.", Bulletin of the American College of Surgeons, vol. 87, no. 11, pp. 12-18, 2002.

[10] R. A. Cherry and J. Ali, "Current concepts in simulation-based trauma education," The Journal of Trauma, vol. 65, no. 5, pp. 1186-1193, 2008.

[11] E. F. J. Block, L. Lottenberg, L. Flint, J. Jakobsen, and D. Liebnitzky, "Use of a human patient simulator for the advanced trauma life support course," The American Surgeon, vol. 68, no. 7, pp. 648-651, 2002.

[12] H. Berkenstadt, Y. Munz, G. Trodler, A. Blumenfeld, O. Rubin, and A. Ziv, "Evaluation of the Trauma-man simulator for training in chest drain insertion," European Journal of Trauma, vol. 32, no. 6, pp. 523-526, 2006.

[13] Virgil, Device developed in simulation program, lauded by army general, http://www.cimit.org/news/virgil.html.

[14] J. Ali, K. A. Ahmadi, J. I. Williams, and R. A. Cherry, "The standardized live patient and mechanical patient models their roles in trauma teaching," Journal of Trauma-Injury, Infection and Critical Care, vol. 66, no. 1, pp. 98-102, 2009. 


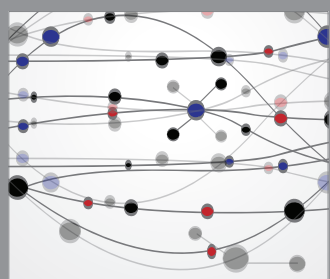

The Scientific World Journal
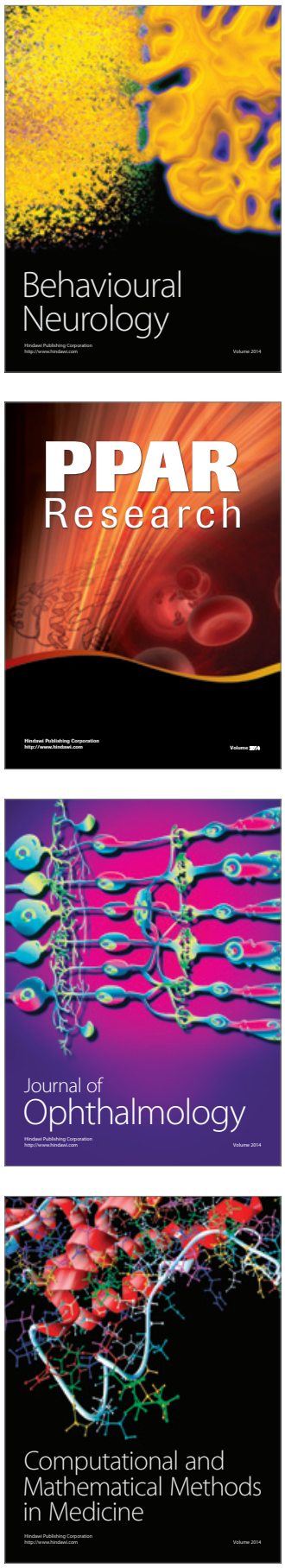

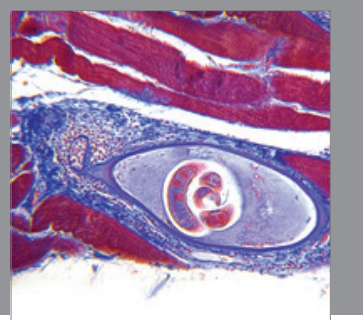

Gastroenterology

Research and Practice
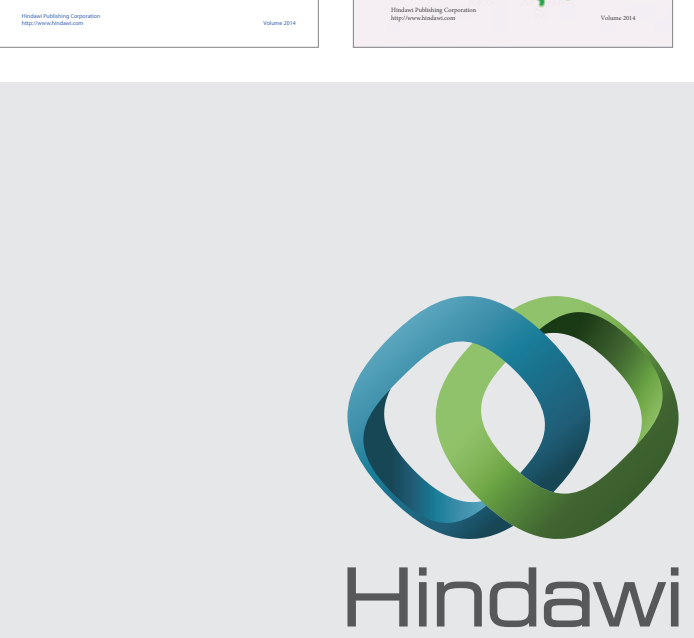

Submit your manuscripts at

http://www.hindawi.com
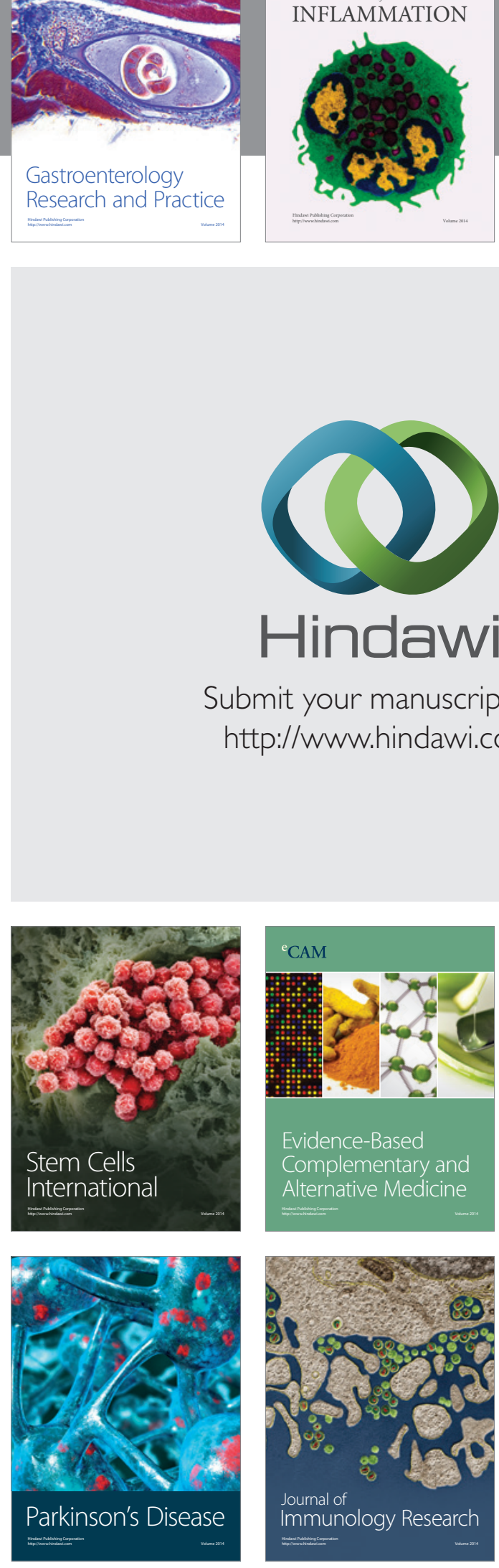

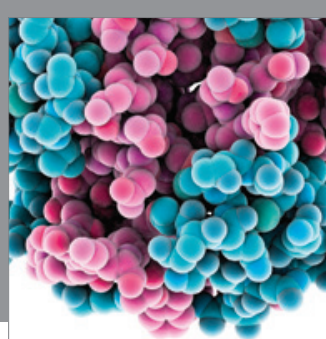

Diabetes Research
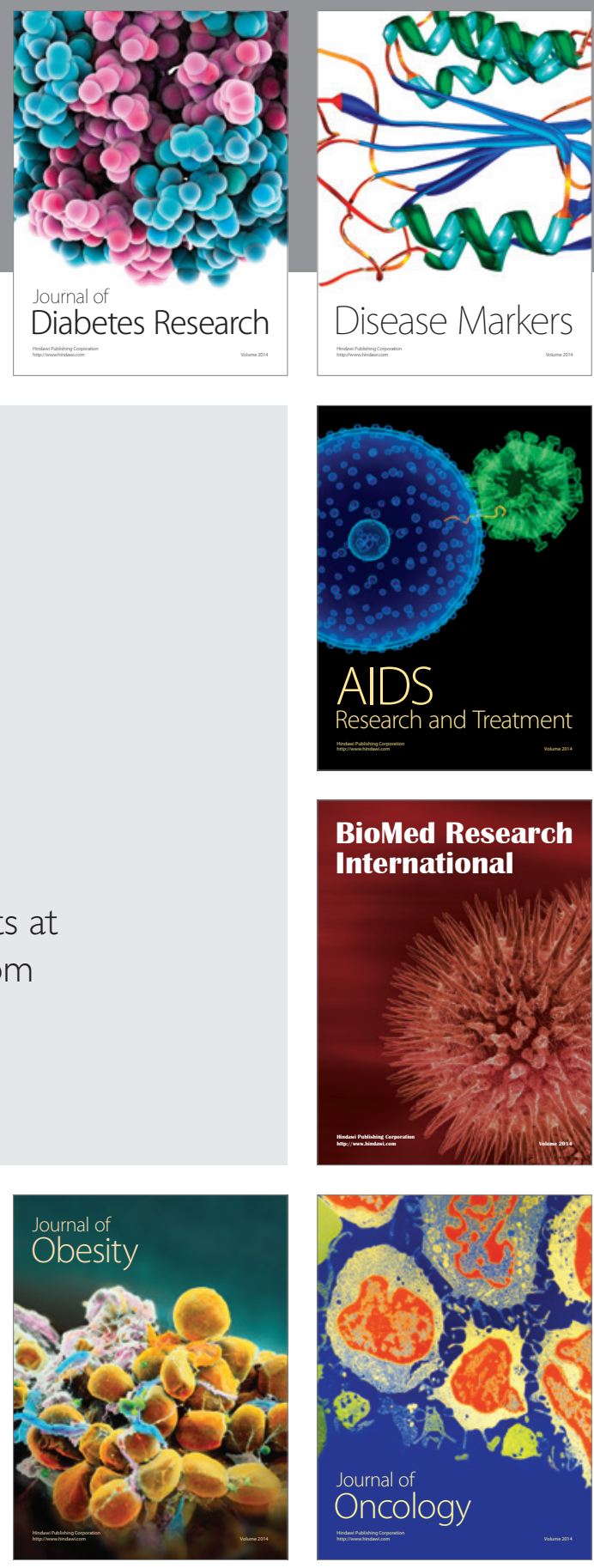

Disease Markers

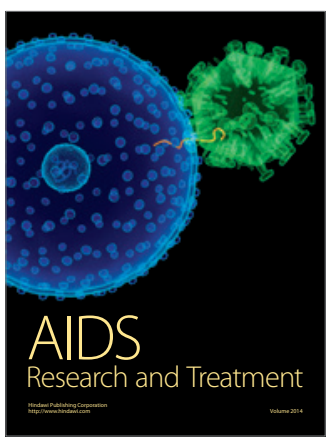

BioMed Research

International
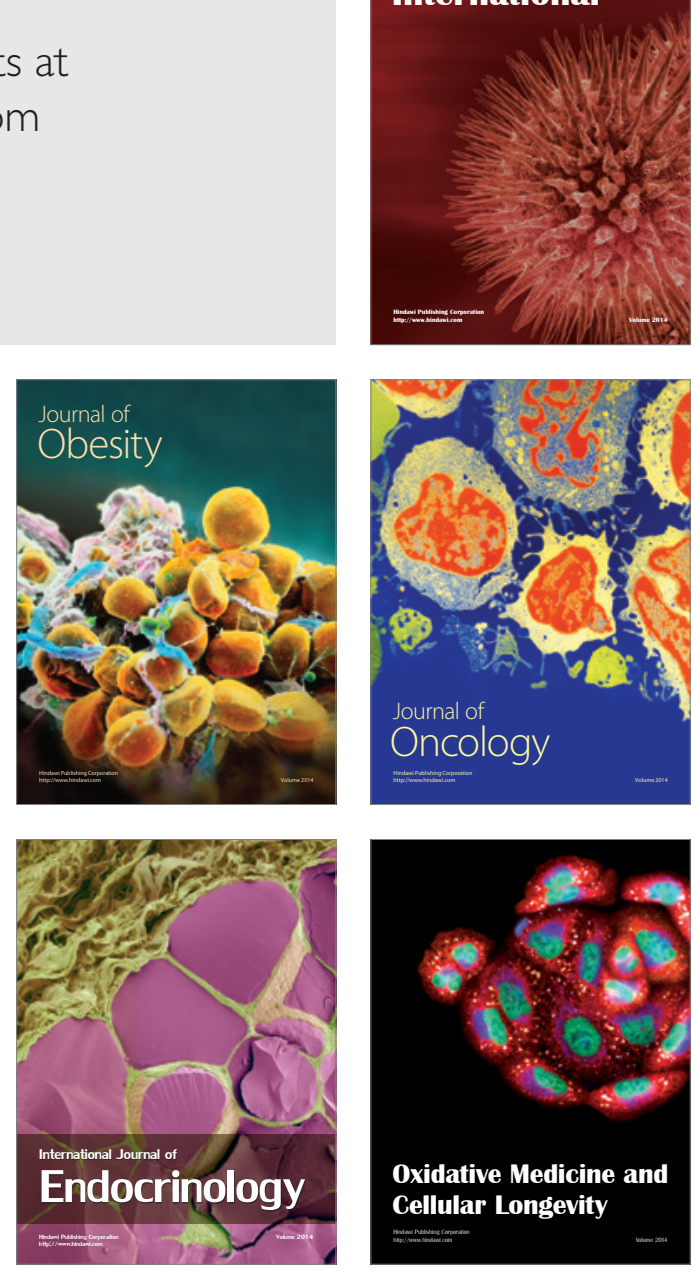